\title{
Geographical perspective of Modeling Primary Healthcare Accessibility
}

\author{
Justice Surage, Richard Tawiah, Timothy Twumasi-Mensah
}

\begin{abstract}
Purpose- The purpose of this study was to measure the spatial accessibility of primary healthcare facility in Ghanaian rural areas, by determining the barriers to health care accessibilities in the Amansie Central district.
\end{abstract}

Design/Methodology/Approach- Both network and proximity analyses were performed on the digitized data such as road networks, settlements, population, district boundary, natural resources (rivers, streams and forest) and site location (health facilities).

Findings- The overall mean distance to the nearest health facility in the district was $8.9 \mathrm{~km}$. Fiankoma sub-district recorded the highest mean distance whereas Tweapease sub-district recorded the least. In general, 31.2\% of the district population has no access to healthcare facility. The Ghana Health Service access criteria that health facility should be accessible to an estimated population within $8 \mathrm{~km}$ radius from the facility were used to ascertain this percentage score. Transportation was identified to be one of the major hindrances to healthcare accessibility and this was as a result of poor road network in the district.

Research limitations/implications- The study was restricted to the Amansie Central District of Ghana. This limits the extent of generalization of results.

Value/Originality- The study proposed additional sites for siting new health facilities base on criteria such as population, distance, centrality and existing infrastructural development. This will consequently improve healthcare accessibility and utilization by increasing total coverage closer to $100 \%$.

Keywords: Accessibility, healthcare facility, population, network, proximity, rural, spatial

Paper type- Research Paper

\section{Introduction}

Healthcare accessibility is widely recognized as an important component of the overall health system. It has the capability of promoting socio-economic growth and development of a nation and reducing disease burdens by improving the quality of physical and mental wellbeing of all. A fundamental principle of health geography is that healthcare services are evenly distributed across space and time and can be easily accessed by the populace. However, in reality, distance to healthcare provider is a significant barrier to healthcare. According to Guagliardo, (2004) and Graves, (2009) healthcare accessibility is affected by factors such as race, ethnicity, language, disability, mobility, distance to health care, and the number of health care providers in an area. Measuring accessibility of healthcare contributes to the understanding of the performance of health systems. The use of Geographic Information Systems (GIS) for the measurement of physical accessibility is well established and has been utilized in many domains including retail site analysis, transport emergency service and health care planning.

Primary health care (PHC) is an imperative strategy to providing "health for all" and is extensively accredited as a universal solution for improving population well-being in the world (WHO and UNICEF, 1978). PHC is essential as it is the most cost-effective method of 
delivering affordable healthcare; PHC has the ability to prevent diseases and decrease health inequality on a large scale in society if it is equitably distributed (Guagliardo, 2004). Despite a decade of trials of various strategies for achieving 'Health for All' in the 1980's, research has shown that, in 1990, more than 70\% of Ghanaians were covering a distance of about $8 \mathrm{~km}$ to access healthcare in the rural areas (Ministry of Health, 1998). As a result, rural infant mortality rates doubled as compared to urban rates. Improving access to healthcare delivery therefore remains a central goal of health sector reform. The Government of Ghana made it a policy to improve access and equity to essential healthcare facilities and ensured that the health sector played an essential role in the National Poverty Reduction Strategy. In 2005, the Ghana Health Service developed a strategic objective which includes improving geographical access to primary and emergency health services by placing a Community Health Officer at strategically identified Health Points in remote areas known as Community-based Health Planning and Services (CHPS) concept (Ghana Health Service, 2005).

The Community-based primary health car has been notified to be the effective way of improving access to health facilities and reducing large inequities in coverage of health services (Ijumba, et al., 2004). Societal, socio-demographic and behavioral factors affecting utilization of PHC services have widely shown that geographical accessibility has a direct bearing on the utilization of health services (Tanser et al., 2006). Distance to health facilities has been associated with maternal and infant mortality (Frankenberg, 1995; Reyes et al., 1998; Thaddeus \& Maine, 1994; van den Broek and Nsowah-Nuamah, 2004). Predictably, improving geographical access to PHC has a direct potential on reducing adverse health outcomes (Perry \& Gesler, 2000; Connor et al., 1994).

Amansie Central District in the Ashanti Region is one of the districts in Ghana which is saddled with socio-economic deprivation and poor health status. The district has 206 settlements with over eighty percent (80\%) of the settlements being rural. There is a prevalence of Buruli ulcer and other diseases such as tuberculosis, malaria, diarrhea, among others (District Health Annual Report, 2010). The staff working in the nine (9) public and private health institutions together with some Non-Governmental Organization for example Health Foundation Ghana has made it possible to improve the health status of the people in the district. The institutions comprise of six (6) governments, two (2) Mission and one (1) private facility.

The rapid increase in uptake of information technologies in the health sector coupled with the current trends with the need to use technology to quantify health outcomes has increased the demands and access to these health facilities. Basically, the district uses the supply ratio method for measuring access to health care. This method indicated that the Out Patient Delivery (OPD) attendance in the district has increased tremendously (The District Health Annual Report, 2010). For the example, Numereso Health Centre which was previously known to have ten OPD visits per week now records twenty visits per week (The District Health Annual Report, 2011). The morbidity and mortality rates have been increasing and decreasing respectively. Maternal and infant health indicators are improving due to much access to safe motherhood services and Expanded Programme on Immunization (EPI) services. In spite of this improvement, maternal mortality is being recorded in the district annually since 2005 (District Health Annual report 2007/11).

According to Shrestha (2010), access can be described in five dimensions; namely availability, accessibility, affordability, acceptability and adequacy. The first two dimensions 
are spatial in nature and can be modeled. Availability refers to the total number of services from which users can make their choices. Accessibility is related to travel impediment (time or distance) between spatial location of user and services. The last three dimensions are not spatial related and have received more attention in Ghana. They relate to cost, cultural factors and perception of health care. According to the District Health Annual Report (2011), culture was not a barrier to health care delivery in the district since majority of the populace share similar culture and also understands health delivery system. Community participation in outreach programmes has always been encouraging. Government has made health care affordable as a result of implementation of the National Health Insurance Scheme (NHIS). According to National Health Insurance Annual Report (2011), 88.5\% of the total district population is enrolled on the scheme of which $75 \%$ are people from rural areas. The resources in the existing health facilities located in the rural areas are capable of meeting the primary health care needs of the populace but the facilities are underutilized. Moreover, people in some communities continue to petition the Government to site health facilities which will be accessible to them. This implies either accessibility being the second dimension of access was not considered during the allocation of these existing health care facilities and or availability which is the first dimension, needs consideration. Thus, new health facilities need to be sited or improving on the existing facilities.

Studies such as Fitzpatrick, et al., (2004), among others indicated that spatial distributions of health services have influence on its accessibility and utilization. Equitable distribution of health care facilities and its resources can potentially contribute to equal information leading to the understanding of epidemiology and etiology of diseases. GIS technology has the potential to address this issue by identifying the best locations for siting health facilities taking into consideration distance to be covered and accessibility of the road network among others. The district and national goal of meeting Millennium Development Goal (MDG's) 4, 5 and 6 is at risk if distance continues to be a barrier to health care.

This study aimed at measuring spatial accessibility of health facilities, estimate population without access to health care and propose appropriate sites for siting functional and accessible health center in the Amansie Central District.

\section{Background and Location of Study Area}

This study was conducted in the Amansie Central District located at the southern part of the Ashanti Region in Ghana. The District is one of the twenty-seven (27) administrative districts in the region. It has about 206 settlements with Jacobu as the Administrative capital which is about $60 \mathrm{~km}$ away from the regional capital (Kumasi). The population consists of about 80\% Akan speaking people and the others such as Ewe, Dagbani, Frafra and dagali. The district populations are located in peri-urban areas (9.3\%) and the rest (90.7\%) are in rural areas. The area is surrounded by hard boundaries in the form of large perennial rivers, forestry areas, and commercial farmland. The district is located within the forest dissected plateau region with an average height between 150 metres and 300 metres above sea level. Topographically, the area is relatively flat with occasional undulating upland of 240 metres to 300 metres above sea level. The district can be found within Latitude $6000 \mathrm{~N}$ and $6030 \mathrm{~N}$ and Longitudes $1000 \mathrm{~W}$ and $2000 \mathrm{~W}$. The total area of the district is about $710 \mathrm{sqkm}$.

It shares common boundaries with Bekwai Municipality formerly Amansie East to the north east, Amansie West to the west, Obuasi Municipal Assembly to the south east, Adansi North to the east, Adansi South to the south and Upper Denkyira in the Central Region to the south. 


\section{Healthcare Delivery in the Study Area}

The district has been divided into five (5) sub-districts with the total staff strength of one hundred and fifty (150). The Ghana Health Service operates $63.64 \%$ of all health facilities in the district. Jacobu sub-district has the highest number of facilities (36.36\%) with Numereso and Tweapease sub-district having the least (2\%) each. In addition, there are two (2) Reproductive and Child Health (RCH) Units and 98 outreach points. These outreach points are visited at least once in a month by public health staff in the district. The challenges of healthcare delivery in the study area are numerous as a result of high proportion of the population located at the rural areas and the poor road network which need to be addressed.

Figure 1 below shows the Amansie Central district map, indicating various settlements and the health facilities. 


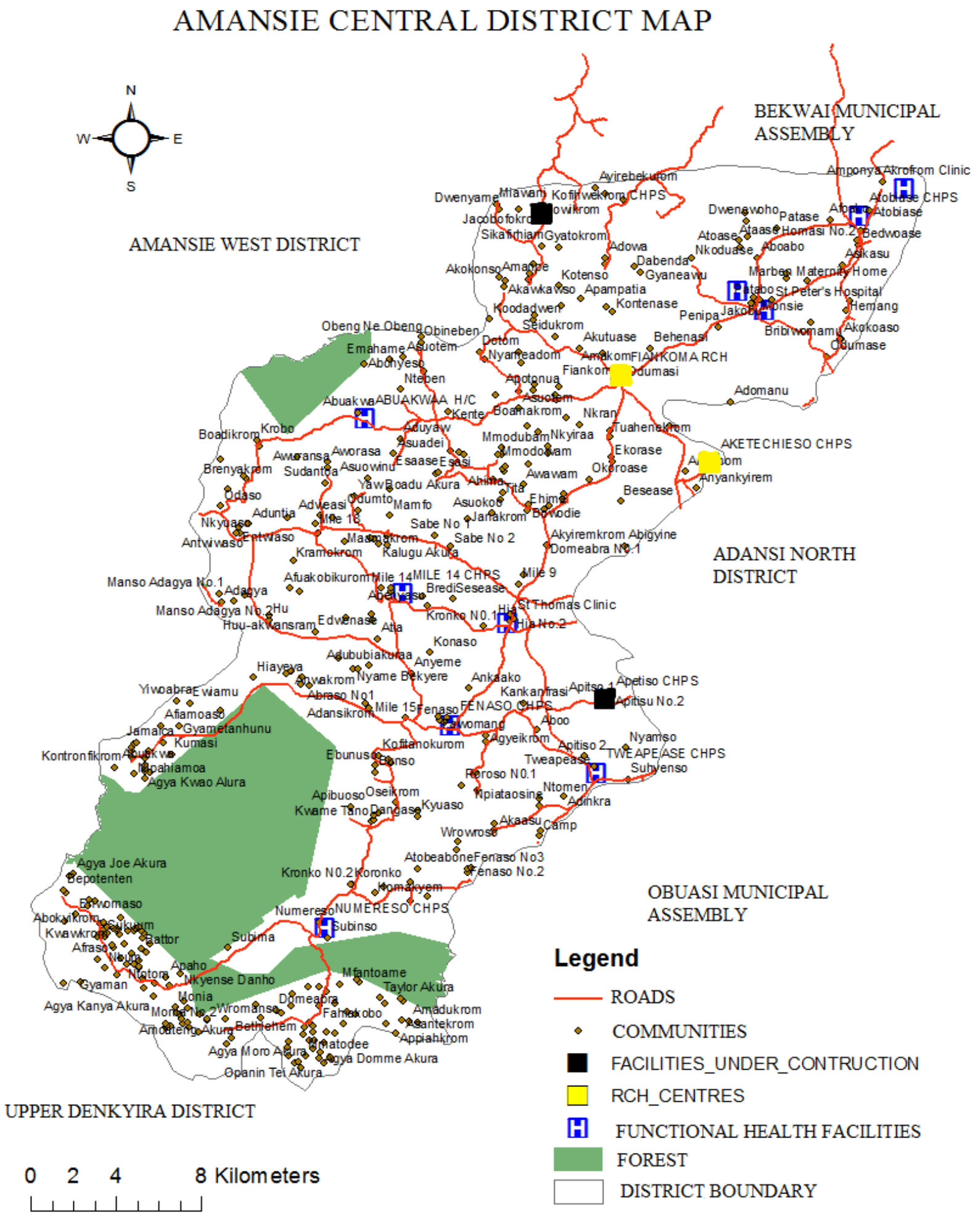

Figure1: Location of Health Facilities and other Spatial Features in the Amansie Central District. 


\section{Materials and Methods}

The major material for the measurement of spatial accessibility to primary healthcare services was the base map of the study area. This was obtained from the Centre for Remote Sensing and Geographical Information System (CERSGIS). The rationale for modeling primary healthcare accessibility in the rural communities was to locate health service nearer to communities and to improve its accessibility. The GIS method employed made it possible to determine populations who have limited access to healthcare services. The Chi Square Goodness of Test was used to the test significance of the descriptive data whereas graphs and tables were also used to display the outcomes of the results.

\section{Study Design}

The research design for this study was a descriptive and exploratory study which was analyzed through qualitative methods. Healthcare facilities in the study area were not located on the base map of the District. With the aid of Global Positioning System (GPS) tool, the coordinates of all the health facilities were taken and inputted in ArcGIS 9.3 software for analysis. The network analyst was used to generate approximate polygons around each health facility in the district. As a result, fourteen polygons were generated showing the serviceable area of each health facility. Observation, face-to-face interviews and Questionnaires were used as data collection methods to collect data to evaluate communities that fell outside all polygons generated. Also, the distances between the communities and the nearest healthcare facilities were measured from the map using both Network and Proximity Analysis in ArcGIS 9.3. The two measure of analysis that is: Network and Proximity Analysis were used in order to ascertain the best measure of spatial accessibility to health facilities. A descriptive statistical method was used to analyze the data.

\section{Data Sources}

The data for this study were both primary and secondary.

i. Primary data: the nearest distance between each community and the health facility was measured in kilometers from the based map with the help of the measuring tools in the ArcGIS 9.3 software. Global Positioning System (GPS) was used to obtain the coordinate of all healthcare facilities in the district. Visual inspection and face to face interview were also applied on the sample of the populations that fell outside the $8 \mathrm{~km}$ kilometer range from the existing health facilities.

ii. Secondary data: The Population of each Community, Number of Health Facilities and Electronic Data such as digital road networks, forest and water bodies of the study area were obtained from the CERSGIS and the Amansie Central district health directorate.

\section{Measuring Spatial Accessibility}

Travel impedance to nearest provider was the method applied in the measuring of spatial accessibility. The distance between the patients' place of settlement or the central point of the community and the nearest health facility were taken into consideration with the motive of ascertaining the distance that patients travel in order to seek for healthcare in the district. 


\section{Sampling Techniques}

The communities that fell outside $8 \mathrm{~km}$ service area of each health facility in the district were randomly selected using simple random sampling techniques. This was carried out by listing the affected communities on pieces of paper which were then mixed up and six samples were randomly selected. These six selected communities were regarded as strata since they are homogeneous. Proportional allocation technique was used to draw a sample of thirty respondents from the selected communities.

\section{Chi-Square Test}

The research hypothesis was that: distance and transport to healthcare centers has no influence on its accessibility and utilization. In the case of this study, it was assumed that all the facilities which were over 8km away from settlements/communities are underutilized due to its inaccessibility. A survey was carried out of the affected communities and the chi-square goodness of test was used to analyze the data at $5 \%$ level of significance. The chi-square is a statistical test that measures the extent to which a set of observed frequencies of a sample data deviates from the corresponding set of theoretical or expected frequencies of the sample.

\section{Proximity Analysis}

A buffer is a zone of specified distance around a selected map feature. A buffer of $8 \mathrm{~km}$ of radius was generated around each health facility. The buffer/circle generated did not consider road networks but rather measured access in a straight line from the health facility. A community that falls outside the buffer zone was classified as a community without access to health care facility in the district. This method is basically used in measuring physical accessibility of health facility in Ghana (Ministry of Health, 1978).

\section{Network Analysis}

Network analysis is the analysis of networks through network theory, generally, graph theory. The network analysis is more objective and scientific than the proximity analysis. This was also used to generate approximates polygon around each facility. What it did was to connect all the communities that lie along the $8 \mathrm{~km}$ distance from the health facility. The lines connecting the various communities are the main road networks. The results from both the field work and based map were compiled and plotted into tables, graphs and maps using computer software such as SPSS, Microsoft excel and ArcGIS.

\section{Results}

Table 1 describes the main variables required in the measurement of spatial accessibility. The descriptive statistics of the data obtained from District Health Directorate, interviews and the based map on the various variables under study were computed. Out of the 206 communities in the District, 204 communities could be traced from the District based map. The remaining two (2) communities could not be traced. 
Table 1: Variables studied

\begin{tabular}{|l|l|l|}
\hline Community & Road Network & Health Care Facility \\
\hline name & nature of road & type of facility \\
distance to Nearest Facility & type of road & categories of staff \\
& mode of transport & \\
\hline
\end{tabular}

There are 14 Health Care Facilities in the district. Two (2) of these facilities namely, Apetiso and Kowikrom CHPS compound were under construction. The researcher's checks indicated that the Fiankoma CHPS Compound was initially in full operation however, due to inadequate staff and equipment, the Fiankoma CHPS Compound currently performs only public health activities. About eighty-two percent (81.8\%) of the health care facilities are being managed by nurses of all categories and $18.2 \%$ are managed by doctors. The district as a whole is classified as a rural district and the only hospital which serves as a referral centre is located in the district capital. The distance between the nearest healthcare facility and the referral centre is $8.60 \mathrm{~km}$.

The computed average distance that a patient travels to seek for healthcare for the entire district was $8.5 \mathrm{~km}$ with a standard deviation of $5.72 \mathrm{~km}$. The network analysis performed gave the following results: $68.76 \%$ of the total population in the district comprising $52.45 \%$ of the total communities have access to health care facilities whereas proximity analysis resulted $96.1 \%$ of the population have access to health facility. This implies that from the results of network and proximity analysis, $31.24 \%$ and $3.9 \%$ of the entire district population respectively, do not have access to healthcare facility. The results of network analysis were preferable to that of the proximity analysis since it took all road networks into consideration, without considering only a straight distance from health facility. The result of network analysis makes it possible to identify new site where additional health facility can be put up to improve accessibility of primary health care services in the district. 


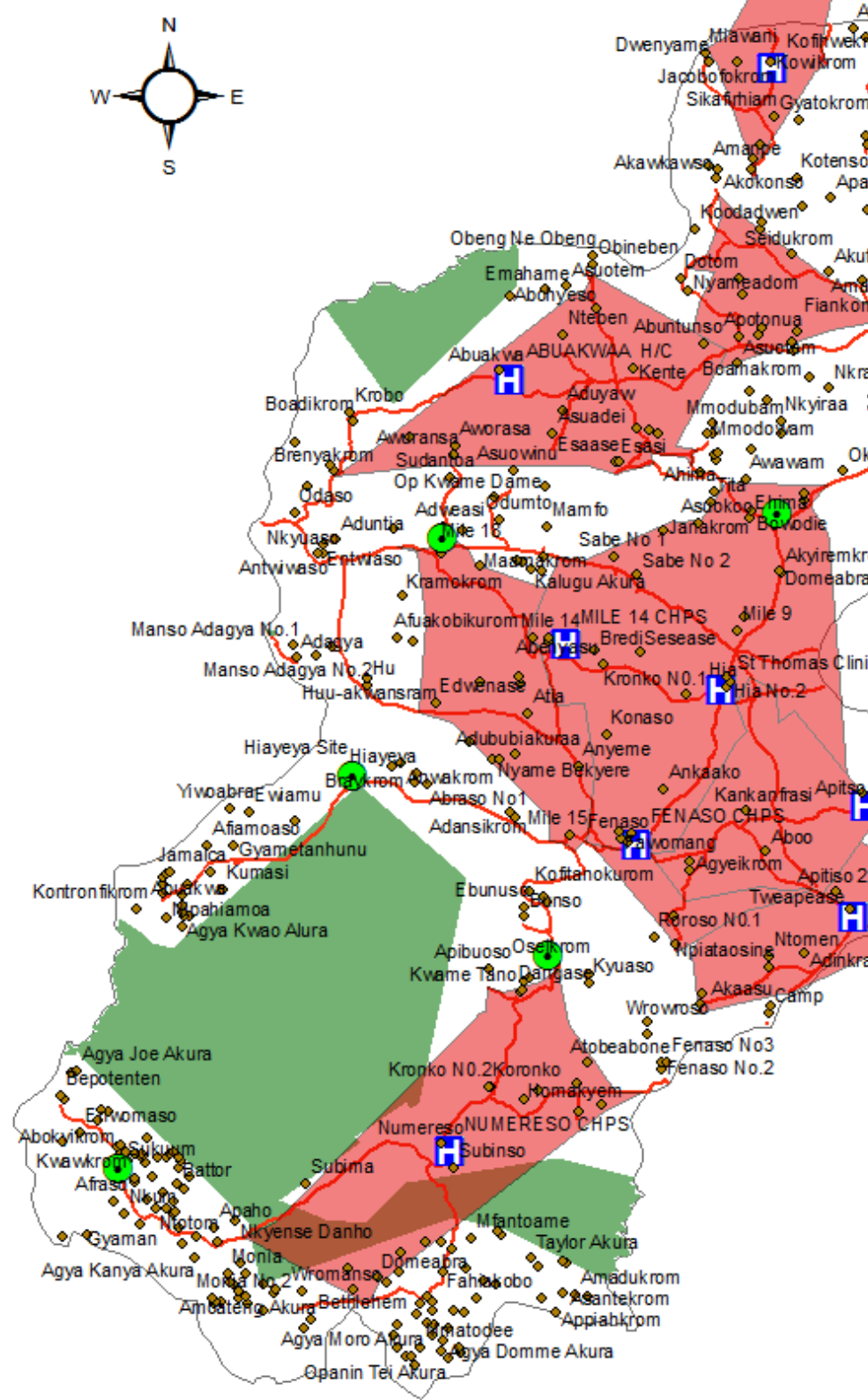

\author{
Legend \\ Proposed sites \\ - communities \\ H Existing Facility
}

\title{
Service A rea
}

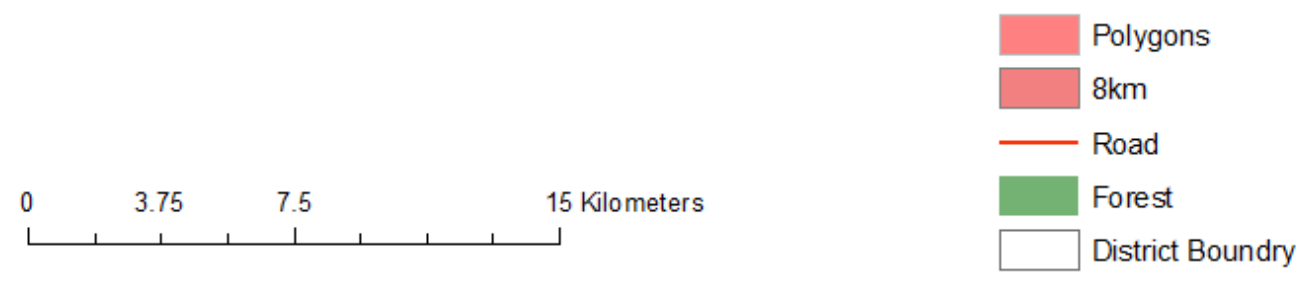

Figure 2: Approximate polygons around health facilities, Amansie Central District 


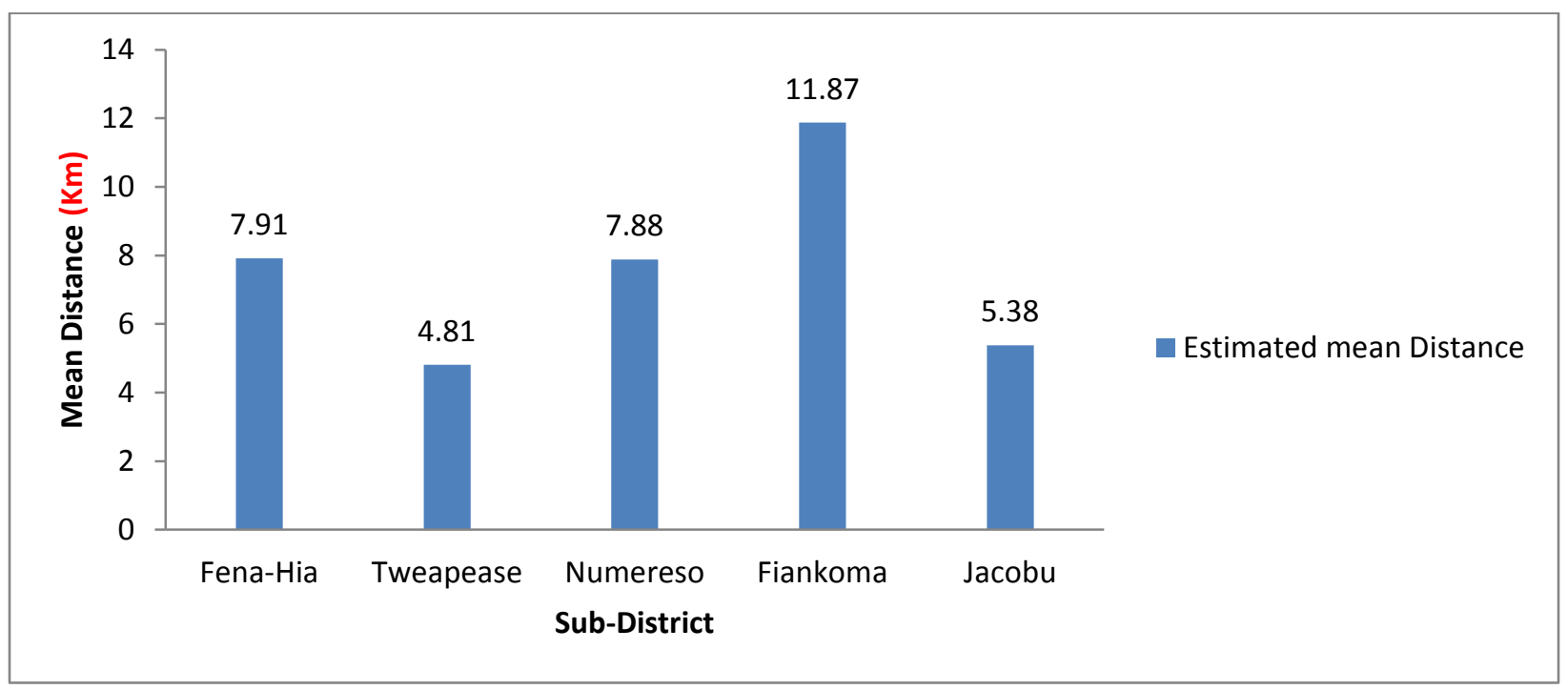

Figure 3: Estimated Mean Distance to the nearest Health Facility by Sub-District

Figure 3 shows mean distances to the nearest health facilities against sub-districts. Fiankoma sub-district recorded the highest mean distance to provider, whereas Tweapease Sub-district recorded the least.

Two variables; distance and transport were considered in the testing for the research hypothesis. How far and or closer a facility is to a patient determines its usefulness. And almost all the respondents agreed to the fact that distance and transport to health facilities have effect on its accessibility and utilization. Exactly 57\% of the respondents interviewed were female and the rest were male. Their ages ranged from 20 to 72 years with the mean age of 36.7 years. Table 2 results display the results of chi square goodness of fit test for transport and distance to health facility

Table 4: Observed and Expected values with Chi Square goodness of fit Test for transport and distance to health facility

\begin{tabular}{|l|l|l|l|l|}
\hline & Observed & Expected & Test statistic & Value \\
\hline transport & 6.0 & 15.0 & Chi-square & 10.8 \\
\hline distance & 24.0 & 15.0 & Probability (p) & 0.01 \\
\hline total & 30.0 & & & \\
\hline
\end{tabular}

The table 2 depicts the result of the interview conducted. Majority of respondents were of the view that access to health facility is mostly affected by distance than transport. Therefore, distance should be considered when spatially distributing health facility. The Chi-square value of 10.8 with corresponding $\mathrm{p}$ value of 0.01 is significance at 0.05 levels. This suggests that distance and transport have different effects on health care accessibility and utilization. From Table 2, it is 
observed that distance to health facility contribute much to inaccessibility and underutilization of the health facilities than transport. Since distance is known to be a good measure of spatial accessibility, we can confidently say that spatial distributions of healthcare services have influence on their accessibility and utilization. Therefore, distance between the communities and the facilities should be considered when allocating health facilities.

Almost all the road networks in the district are feeder roads with the exception of the 5.6km road network that link the district capital to the Kumasi-Obuasi highway and the communities along the Kumasi-Obuasi-Dunkwa highway. The roads are narrow, dusty, and very slippery especially during rainy season. These conditions have serious effects on emergency medical service delivery and outreach programs. The nature of roads in the district has contributed to frequent breakdown of vehicles, motorbikes and worse of all accidents (District Health Annual Report, 2011). Interactions made with the indigenes disclosed that, the inadequate public transportation system in the district was due to the poor road network. The nature of the roads discouraged commercial vehicles from operating in the district for fear of frequent breakdowns and repairs. This situation has compelled most patients to travel on foot to access health care.

Lack of access to affordable transportation has been identified to be a major contributor to health disparities. As a result, families spend a large percentage of their budgets on transportation and other expensive options at the expense of other needs including health care (Thoen, 2010). The result of the interviews conducted and observations made revealed that approximately $80 \%$ of patients depend on public transport to access health care services. Only few commercial vehicles are operating in the rural communities in the district and these are mostly over loaded to the extent that passengers sometimes have to sit on the carriers of the vehicles. The negative impacts of inadequate access to transportation on health were not fully considered in this study due to inadequate data and resources. However, a reasonable number of (researchers) have connected access to healthcare facilities and health outcomes with transportation. Results indicated that most vulnerable populace such as those with some forms of disabilities and the elderly can have a pronounced impact on their overall well-being due to lack of transportation options (Fitzpatrick et al., 2004). 


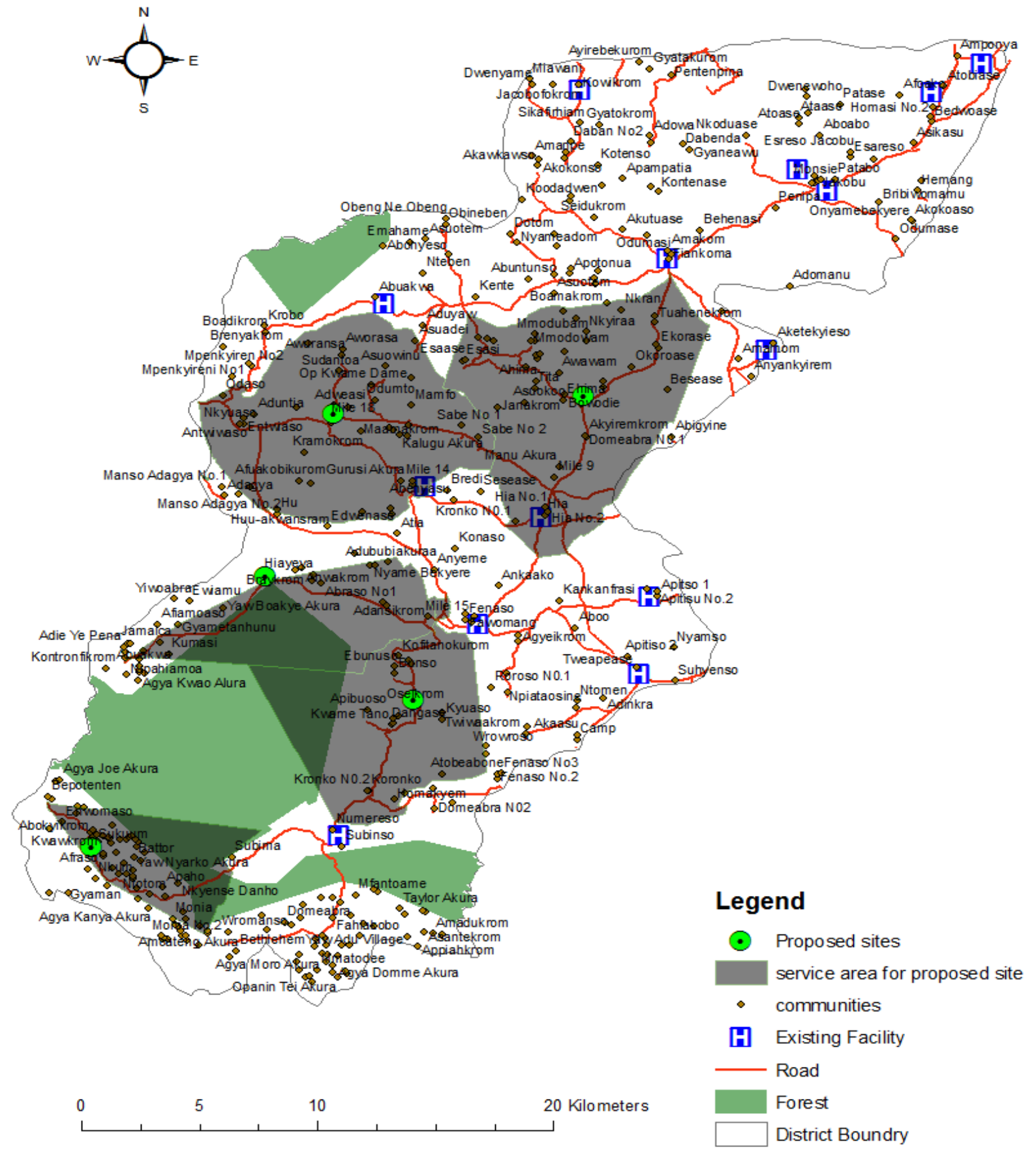

Figure 3: Approximate polygons around proposed sites, Amansie Central District. 
In order to improve the health status of the people in the district by ensuring access to the healthcare facilities, the study identified five locations for siting new health facilities. These areas include: 1. Oseikrom, 2. Mile 18, 3. Hiayeya, 4. Nankawora and 5. Sukuumu. Should the health facilities be located here, they would potentially deliver healthcare services to a greater number of people in the district (See Figure 3). The proposed sites have a total of 80 communities with a population of about 29213 (that is $30 \%$ of the district population). Currently, patients in these communities indirectly have no access to healthcare facility since they cover more than $8 \mathrm{~km}$ to access healthcare. On the average, patients in these areas cover not less than $14.54 \mathrm{~km}$ to access health care services. The poor nature of road network in these areas has increased transportation cost.

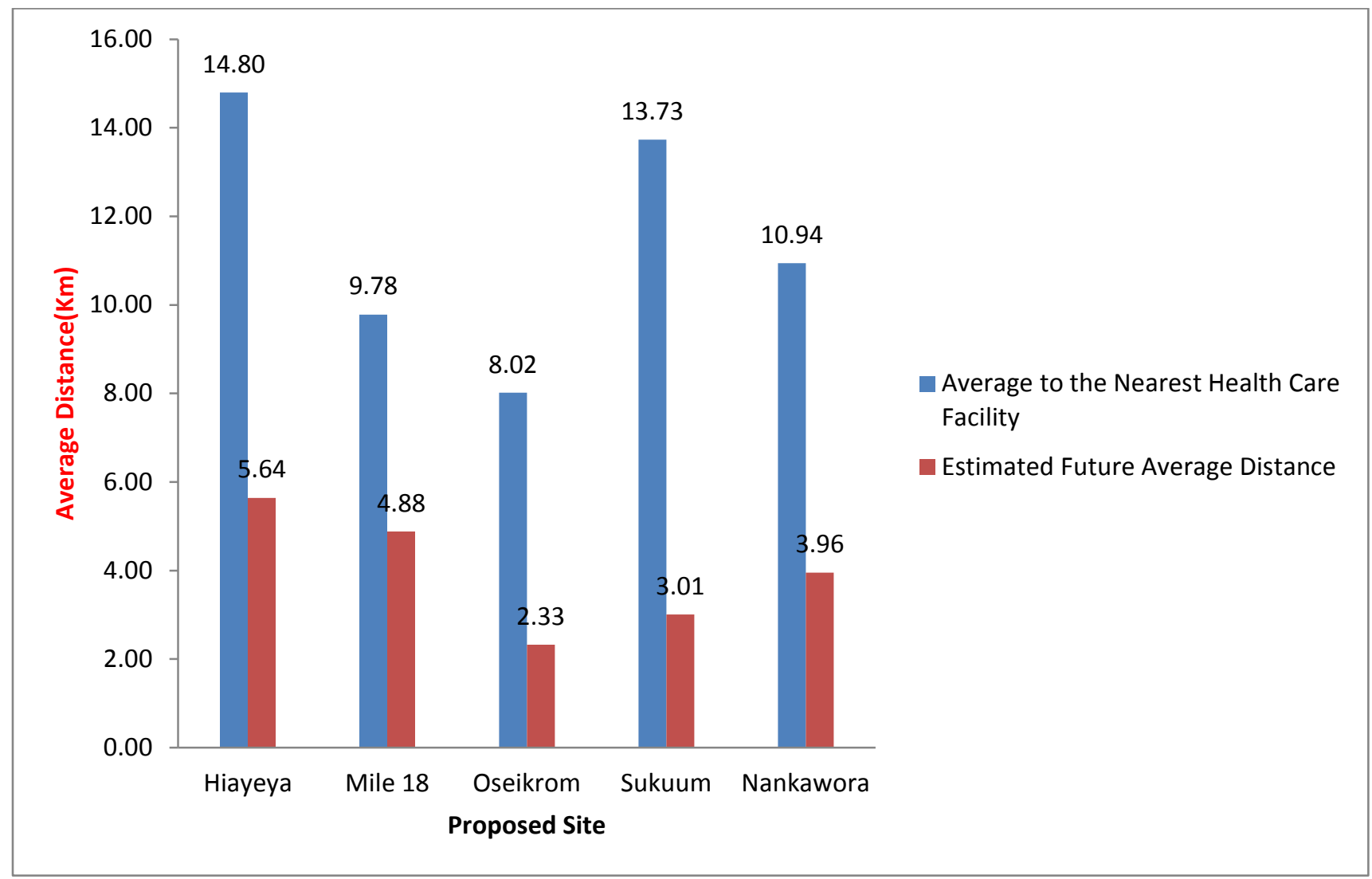

Figure 4: Distribution of Average Distances by proposed sites

In Figure 4, the study compares both current and future average distances within catchment area of the proposed sites. The chart indicates that the population within Hiayeya catchment area currently travels on the average distant of $14.8 \mathrm{~km}$ to seek healthcare services but in future if a facility is placed at the proposed site, then the distance would reduce to $5.64 \mathrm{~km}$. This shows that the population within the catchment area of the proposed sites will no longer suffer from distance to seek healthcare services. 


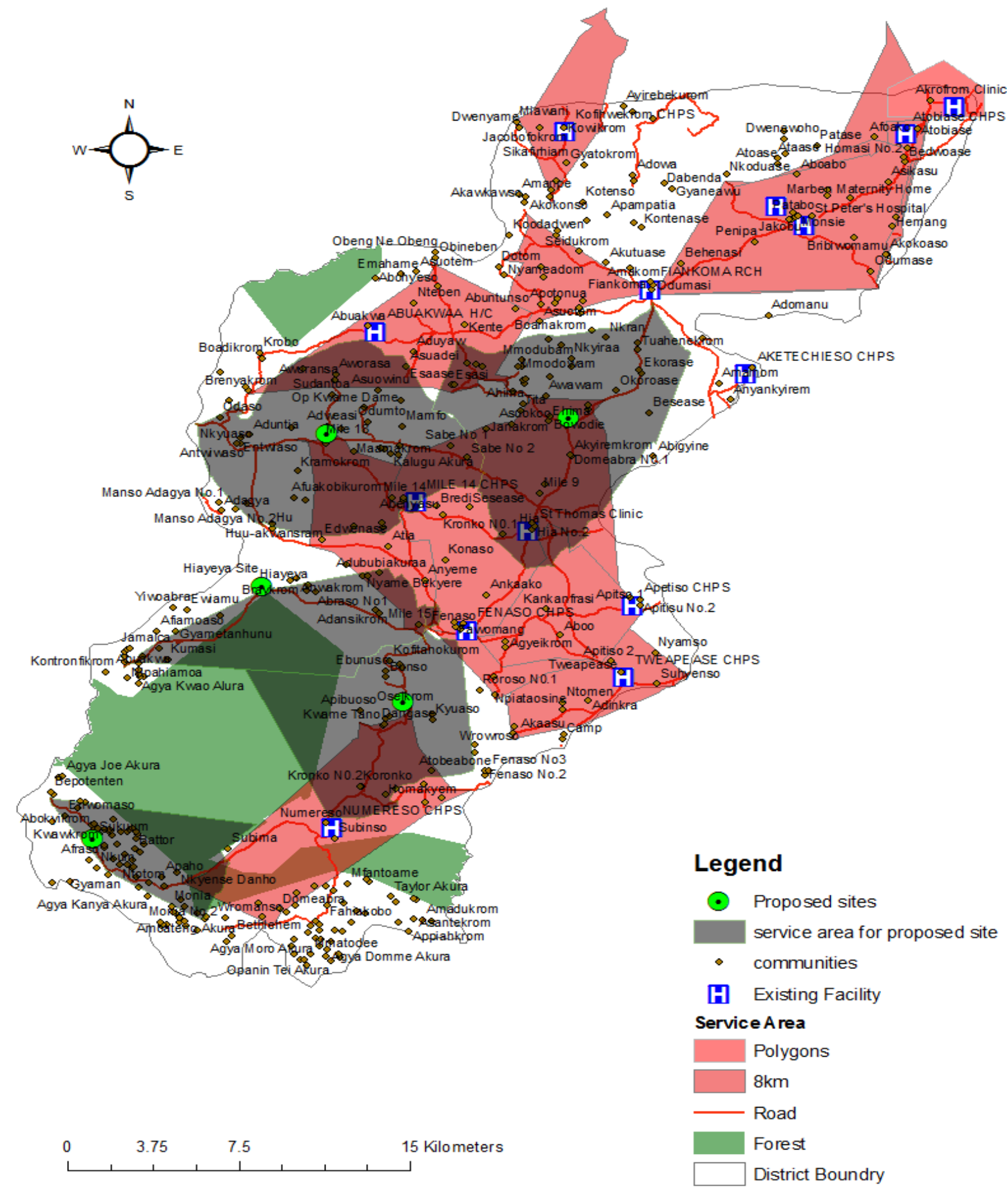

Figure 5: Existing and proposed health facilities in the Amansie Central District.

Figure 5 shows the serviceable area of both existing health facilities and the proposed sites. Even though their boundaries overlap, the analysis shows that $10 \%$ of the district population will not have access to any health facility. 


\section{Discussion}

Accessibility simply refers to how easy it is to get to a site, and can be measured in terms of travel time or distance. Examining accessibility can help to determine whether a facility would achieve its intended purpose and also help in decision making. People in rural areas have been denied social amenities such as electricity, good road, safe and reliable water supply, education among others. The provision of a health care facility becomes beneficial if it is accessible. Primary health care (PHC) can potentially assist in this direction. According to Guagliardo (2004), PHC is the key in preventing diseases and decreasing health inequality on a large scale in society if it is equitably distributed.

The proximity analysis and network analysis were the main spatial measuring approaches considered in this study. Results from the proximity analysis revealed that $3.9 \%$ of the district populations have no access to any health facility whereas network analysis indicated $31.24 \%$. The study considered network analysis measure of spatial accessibility since it considered the facts that, road networks are not always in straight lines.

On the average, patients in the district travel a minimum of $8.5 \mathrm{~km}$ and a maximum of $38 \mathrm{~km}$ with a standard deviation of $5.7 \mathrm{~km}$ to access health care. Fiankoma sub-district recorded the highest mean distance of $(11.9 \mathrm{~km})$ while Jacobu sub-district recorded the least $(4.8 \mathrm{~km})$.

Transport was known in the district to be the major barrier to access health care facilities. This research also disclosed that distance to health facilities is the main hindrance to accessibility and utilizations. Majority of the respondents were of the view that though transportation is a challenge, if the distance to health facility is shorter it may perhaps promote travelling by foot. The problem of transportation was as a result of the poor road network. The roads are eroded and muddy in nature making movement more difficult during rainy seasons. Additional sites have been proposed base on criteria such as population, distance, centrality and existing infrastructural development which can have the potency to improve spatial access to health care. Not all proposed sites necessarily required well established Health Centre. Some could be outreach point due to inadequate resources. For instance, Nankawora and Oseikrom sites could be outreach points. The populations within the catchment area of the proposed sites travel a minimum mean distance of $8.02 \mathrm{~km}$ to the nearest facility and maximum of $14 \mathrm{~km}$. The population within the catchment area of these sites, on average, spend GH4 7.00 on transportation on a daily visit to access health facility. Also effort should be made to complete the construction and rehabilitation works which are currently on going in Fiankoma, Apitiso and Kofihwekrom communities as they can potentially improve accessibility.

\section{Conclusion}

The rationale for the study was to apply Geographical Information System (GIS) technology to measure access to primary care facility in the district. It was realized that health service in Ghana is mostly measured in terms of Provider-to-Population Ratio method commonly known as supply ratio. The method does not necessarily require GIS application Guagliardo (2004). This makes it important to apply GIS in this study since it is known to be applied on similar work by renowned 
researchers such as Connor et al., (1994), Lang (2000), Andean et al., (2000), Kofie \& MollerJensen (2001), Oliver \& Mossialos (2004), Nasser et. Al., (2005), Najeeb (2010), Mallick \& Routray (2001) and others. It is always important to measure access to health facility geographically than the supply ratio since it has the capacity to ensure uniformly distribution of facilities. The average distance a patient travel to seek for healthcare services was $8.9 \mathrm{~km}$. The minimum distance was $8.5 \mathrm{~km}$ and the maximum was $38 \mathrm{~km}$. With this, $31.24 \%$ of the district populations were identified to have no access to health facility given the criteria that health facility should be accessible to population within $8 \mathrm{~km}$ distance from the facility. The $31.24 \%$ of

the populace who were not having access to healthcare did not fall within the frequency polygons. Spatial accessibility of health facilities in the district was about $68.76 \%$. However, the poor nature of road networks leading to poor transport system was a major obstacle to access healthcare services in most of the communities. The study found that, access to healthcare services could improve with periodic maintenance of road network in the district. Also district health authorities could introduce mobile clinic system to supplement the existing health facilities by serving the immediate needs of population without access to health facility, example, Bepotenten community which is $17.34 \mathrm{~km}$ away from the nearest health Centre. Again, government and other stakeholders should put in much effort to speed up the construction and rehabilitation work on the new facilities since those facilities have higher potential of improving access to healthcare. Further, the proposed sites for building new facilities should be considered when the needs arise. The maps composed gave a visual impression about the situation on the ground hence could be of help to policy makers to make informed decision in the near future.

\section{References}

District Health Directorate (2011), “Amansie central district health annual report”, Ghana Health service, Jacobu.

Connor, R. A., Kralewski J. E., Hillson S. D. (1994), “Measuring geographic access to health care in rural areas”. Med Care Rev,51:337-77.

Fitzpatrick, A. L., Powe, N. R., Cooper, L. S., Ives, D., \& Robbins, J. A. (2004), “Barriers to health care access among the elderly and who perceives them”, American Journal of Public Health, 94(10), 1788-1794

Frankenberg, E. (1995), "The effects of access to health care on infant mortality in Indonesia”, Health Transition Review, 5(2), 143-163.

Ghana Health Service (2005), “Community Based Health Planning and Services (CHPS)”, (GhanaHealth Service Policy Document No. 20), Accra.

Graves, A. (2009) “A Model for Assessment Of Potential Geographical Accessibility: A Case for GIS”, Online Journal of Rural Nursing and Health Care, vol. 9, no. 1, Spring 2009.

Guagliardo, M. F. (2004), "Spatial accessibility of primary care: concepts, methods and challenges”, International Journal of Health Geographics, 3:available from: http://www.ijhealthgeographics.com/content/3/1/3 
Ijumba, P., Day, C., and Ntuli, A. (2004), “South African health review 2003/2004”, Durban: Health Systems Trust.

Kofie, R. Y. \& Moller-Jensen, L. (2001) “Towards framework for delineating sub-districts for primary healthcare administration in rural Ghana: using GIS”, NorskGeografisk Tidsskrift Norwegian Journal of Geography, 55, 26-33.

Lang, L. (2000), “GIS for health organisations”, California: ESRI Press, [ISBN 1-879102-65X].

Mallick, R. K. and Routray J. K. (2001), "Identification and accessibility analysis of rural service centres in Kendrapara District, Orissa, India; a GIS-based application”, JAG, 3:99-105.

Ministry of Health of the Republic of Ghana (1998), “A profile of health inequities in Ghana”, Accra, Ministry of Health.

Najeeb, Al-Shorbaji (2010), "Use and potential of geographic information systems for health mapping in the Eastern Mediterranean Region.

Nasser B., George L. B. and Alec H., (2005) "Measuring spatial accessibility to primary health care”, Presented at SIRC : The 17th Annual Colloquium of the Spatial Information Research Centre University of Otago, Dunedin, New Zealand.

Perry, B. and Gesler, W. (2000), "Physical access to primary health care in Andean Bolivia", Social Science and Medicine, 50:1177-1188.

Reyes, H., Tome, P., Gutierrez, G., Rodriguez, L., Orozco, M., and Guiscafre, H. (1998), "Mortality due to diarrhea in Mexico: A problem of accessibility or quality of care", Salud Publica De Mexico, 40(4), 316-323.

Shrestha, J. (2010), "Evaluation of Access to Primary Healthcare: A Case Study of Yogyakarta", Indonesia, Enschede, ITC Msc.

Tanser, F., Gijsbertsen, B. and Herbst K. (2006), "Modelling and understanding primary health care accessibility and utilization in rural South Africa: An exploration using a geographical information system”.

Thaddeus, S., and Maine, D. (1994), “Too far to walk: Maternal mortality in context. Social Science and Medicine”, 38(8), 1091-1110.

Thoen, M.J.J (2010), “Equal access for a healthy community”, Unpublished manuscript, Research report on inequality of accessibility of hospitals in Yogyakarta, bachelor thesis executed at PUSTRAL, UGM Yogyakarta, Indonesia.

Van den Boom, G. J. M and Nsowah-Nuamah, N.N.N. (2004), “Overbosch, G.B: Healthcare Provision and Self-medication in Ghana”, (accessed via Web Archive) http://web.archive.org/web/20070625163825/http://www.saga.cornell.edu/images/vadeb om.pdf (accessed 30 August 2008).

World Health Organization and UNICEF (1978), "Report of the International Conference on Primary Healthcare”, Alma-Ata, World Health Organization. 
